\title{
Effect of the molecular targeted drug, erlotinib, against endometrial cancer expressing high levels of epidermal growth factor receptor
}

Toshio Nishimura ${ }^{1}$, Kazuto Nakamura ${ }^{2 *}$, Soichi Yamashita ${ }^{1}$, Sadatomo $l k e d a^{1}$, Keiko Kigure ${ }^{2}$ and Takashi Minegishi ${ }^{1}$

\begin{abstract}
Background: The epidermal growth factor receptor (EGFR) tyrosine kinase inhibitor, erlotinib, has been clinically applied for the treatment of a variety of tumors with EGFR overexpression. A phase II clinical study of erlotinib (NCIC IND-148) for recurrent or metastatic endometrial carcinoma (EC) resulted in an unfavorable result. However, in that study, the expression levels of EGFR were not accurately analyzed. Thus, the aim of this study was to re-examine the efficacy of erlotinib in EC cells by utilizing in vitro and in vivo models.

Methods: Tissue samples obtained from patients histologically diagnosed with EC of the uterine corpus were subjected to immunohistochemistry and RT-PCR to determine the protein and mRNA expression levels of EGFR. Western blot and WST-1 assays of EGFR siRNA-transfected HEC-1A, KLE, and Ishikawa cells were used to evaluate the efficacy of erlotinib in tumor cell lines expressing different EGFR levels. Furthermore, HEC-1A and Ishikawa cells were implanted into athymic mice treated with either erlotinib or trastuzumab.

Results: At our institution, $20.9 \%$ of endometrial cancer patients with low grade endometrioid histology have been diagnosed as stage III and IV. Immunohistochemical analysis and RT-PCR revealed the presence of significant EGFR and EGFR mRNA expression in low-grade endometrioid carcinoma in comparison with high-grade endometrioid carcinoma. In vitro study, WST-1 assay and Western blot analysis revealed that EGFR expression levels were correlated with tumor cell viability. Erlotinib reduced the proliferation of HEC-1A expressing high levels of EGFR, while trastuzumab showed similar effect in Ishikawa cells dominantly expressing human epidermal growth factor receptor type2 (HER2). In vivo erlotinib decreased tumor growth in mice xenografted with HEC-1A cells, whereas this tumor-growth inhibition was not observed in trastuzumab-treated mice xenografted with Ishikawa cell.

Conclusions: EGF contributed to tumor proliferation in EC cell lines along with EGFR expression in vitro. Erlotinib also demonstrated anti-tumor effects in xenograft mice models. Our results suggest that erlotinib continues to have clinical usefulness in specific cases, after taking into consideration the EGFR expression levels.
\end{abstract}

Keywords: Molecular targeted drug, Erlotinib, Endometrial cancer, EGFR

\footnotetext{
* Correspondence: nkazuto@gunma-cc.jp

${ }^{2}$ Gunma Prefectural Cancer Center, 617-1, Nishimachi, Takabayashi, Ota,

Gunma 373-8500, Japan

Full list of author information is available at the end of the article
} 


\section{Background}

Endometrial carcinoma (EC) is one of the most common gynecological malignant tumors in Japan; over 8000 women were diagnosed with it in 2012. There are two subtypes of endometrial carcinoma according to the clinico-pathological characteristics: type I EC and type II EC $[1,2]$. Recent studies on gene signature in EC cells have reported numerous genetic disorders that initiate carcinogenesis: for instance, PTEN, which regulates normal cell function, is highly mutated in type I EC, whereas $\mathrm{p} 53$, which prevents genome mutation, is altered by up to $80-90 \%$ in type II EC [3].

Type I EC accounts for about $80 \%$ of EC, and is generally associated with better prognosis than type II EC since it is composed of low grade endometrioid histology with less aggressive characteristics and favorable prognosis [3]. However, the number of patients with advanced stage or recurrent low-grade tumors might not be negligible since type I EC comprises about $80 \%$ of the newly diagnosed EC in Western Europe, North America, and Japan [3, 4].

After staging surgery, adjuvant therapy is considered based on the pathological risk factors, such as tumor grade, histological type, myometrial invasion, positive margin, lymphovascular space invasion, and positive node status [5]. Radiotherapy has proved to reduce the risk of local recurrence, but no randomized study has shown benefit for overall survival $[6,7]$. In the last decades, there has been emerging evidence suggesting that systemic cytotoxic chemotherapy may have favorable prognosis in advanced EC $[8,9]$. Taxanes, platinum agents, and anthracyclines have been utilized in advanced and recurrent EC patients, with response rates to these drugs ranging from 33 to $57 \%[8,10-14]$.

Cytotoxic cancer therapy induces apoptosis in cancer cells by inhibiting microtubule function, protein function, or DNA synthesis. In contrast, molecular-targeted therapy is designed to interfere with specific molecules involved in cancer cell proliferation and metastasis. Recently, a better understanding of the molecular and genetic characteristics of EC has promoted clinical research that targets angiogenesis and cellular signaling pathways involved in cancer development and progression. Epidermal growth factor receptor (EGFR) has been shown to be overexpressed in many human cancers, including lung $[15,16]$, central nervous system [17], head and neck [18], bladder [19], pancreas [20], and breast [21]; and it correlates with poor prognosis [22]. EGFR expression has been found to be associated with patient outcomes in $43-67 \%$ of EC tissue samples [23-25] and different profiles in low and high grade EC [26]. Furthermore, some studies have reported that the EGFR family plays an important role in the development of EC $[27,28]$. Collectively, inhibition of EGFR function can be a clinical benefit. Targeted therapy against the signaling system of the tyrosine kinase family could be beneficial for patients with type II EC $[29,30]$. Those reports found that the expression of EGFR and human epidermal growth factor receptor type2 (HER2) in type II EC was 30.5 and $16.5 \%$, respectively, whereas it was 51.5 and $2 \%$ in well (Grade 1, G1) and moderately (G2) differentiated endometrioid cancer, respectively. However, there have been no promising therapies, including small molecule tyrosine kinase inhibitors or anti-EGFR monoclonal antibodies, for antagonizing EGFR functions [31, 32]. Thus, in this study, we aimed to evaluate whether targeting the EGFR tyrosine kinase may have a therapeutic effect against EC, by accurately assessing the expression levels of EGFR in cancer cells.

\section{Methods}

\section{Reagents}

Erlotinib (Abcam, Tokyo, Japan) was dissolved in DMSO, and pertuzumab (Tyugai, Tokyo, Japan) was dissolved in distilled water for the in vitro and in vivo studies. Epidermal growth factor (EGF) (Invitrogen, Carlsbad, CA, USA) was dissolved in phosphate buffered saline (PBS) (stock solution: $20 \mathrm{ng} / \mathrm{mL})$.

DMEM (without phenol red) and gentamicin sulfate (Geneticin) were purchased from Invitrogen (Carlsbad, CA, USA). DMEM/Ham's nutrient mixture F-12 (1:1, vol/vol) (without phenol red) was purchased from Sigma-Aldrich (St. Louis, MO, USA).

\section{Cell culture and culture condition}

Ishikawa cells were purchased from Japanese Collection of Research Bioresources (JCRB) cell bank (Tokyo, Japan). HEC293, HEC-1A, and KLE cells were purchased from American Type Culture Collection (Manassas, VA, USA). All the cells used for the experiments were between the $5^{\text {th }}$ and $20^{\text {th }}$ passages.

HEC293 and Ishikawa cells were maintained in DMEM supplemented with $10 \%$ charcoal fetal bovine serum (FBS) and $50 \mu \mathrm{g} / \mu \mathrm{L}$ gentamicin sulfate. HEC-1A cells were maintained in McCoy's 5A medium supplemented with $10 \%$ charcoal FBS. KLE cells were maintained in DMEM/ nutrient mix F-12 Ham's supplemented with $5 \%$ charcoal FBS. All media used were phenol red free. Cells were incubated at $37{ }^{\circ} \mathrm{C}$ in a humidified atmosphere containing $5 \%$ $\mathrm{CO}_{2}$. All cells were harvested using trypsin/EDTA when confluence was less than $80 \%$. All cells were used all experiments when cell concentration was $60-70 \%$.

\section{Tissues and patient cohort}

Resected tissue samples (only hysterectomy samples without neoadjuvant treatment) were obtained from 51 endometrioid adenocarcinoma patients of Asian descent between May 2007 and March 2011 from Gunma University Hospital. All patients had been identified as uterine corpus endometrioid adenocarcinoma carriers on the basis 
of gynecologic and pathological reports. Staging was defined according to the International Federation of Obstetricians and Gynecologists (FIGO) Surgical staging system [33]. The histological grade classification was according to the World Health Organization classification and FIGO [34]. This study was approved by the Institutional Review Board of Gunma University (Permit Number: 12-49) and conducted according to the ethical guidelines of Gunma University. Tissue specimens were handled according to the local ethics committee guidelines. Written informed consent was obtained from all patients enrolled in the study.

\section{Immunohistochemistry}

Formalin fixed samples were embedded in paraffin and sectioned and dried; then, they were deparaffinized and rehydrated. The sections were immunostained using DAKO ENVISION+ KIT/HRP (DAKO, Carpentaria, CA, USA) and Histofine SAB-PO kit (Nichirei, Tokyo, Japan) according to manufacturers' protocols. Rabbit monoclonal anti-EGFR antibody (diluted 1:100, DAKO, Carpentaria, CA, USA) and mouse monoclonal anti-HER-2 antibody (Santa Cruz Biotechnology, Santa Cruz, CA, USA) were used for immunohistochemistry (IHC) to determine EGFR and HER-2 expression levels. Two independent pathologists who were blinded to the clinical information assessed EGFR and HER-2 expression. The inter-observer disagreements (kappa statistics: 0.91) were reviewed a second time, followed by a conclusive decision by both pathologists. A positive result was defined as cell membrane staining of $50 \%$ or above, independently of intensity.

\section{Western blotting}

Twenty-four hours before starting the analysis, all cells were changed to a free- FBS medium. For the analysis of phosphorylated extracellular signal-regulated kinases (ERK) $1 / 2$, cells were treated with EGF (range from $1 \mathrm{pg} / \mathrm{mL}$ to $1 \mathrm{ng} / \mathrm{mL}$ ) for $10 \mathrm{~min}$, washed twice with cold PBS, and incubated on ice with RIPA buffer $(\mathrm{pH} 7.4$, supplemented with protease inhibitors, $200 \mathrm{mM} \mathrm{NaF}, 200 \mathrm{mM}$ sodium orthovanadate) for $30 \mathrm{~min}$. Lysates were aspirated and centrifuged at $15,000 \mathrm{rpm}$ for $10 \mathrm{~min}$ at $4{ }^{\circ} \mathrm{C}$. The protein concentration was measured in the collected supernatant. Frozen patient samples were homogenized and lysed in RIPA buffer. Protein samples (10-20 mg) were diluted in equal volume sample buffer $(\mathrm{pH}$ 6.8, 4 \% SDS, $10 \% 2$ mercaptoethanol, $20 \%$ glycerol, $0.004 \%$ bromophenol blue, $0.125 \mathrm{M}$ Tris- $\mathrm{HCl}$ ) and incubated for $30 \mathrm{~min}$ at $25{ }^{\circ} \mathrm{C}$. Protein samples were loaded on a $12 \%$ polyacrylamide/ bisacrylamide SDS-PAGE gel and transferred onto a PVDF membrane (BIO-RAD, Hercules, CA, USA). Membranes were blocked with $5 \%$ BSA or $5 \%$ skim milk in TBST (100 mM Tris, $0.9 \% \mathrm{NaCl}, 0.1 \%$ Tween-20, pH 7.4) for $1 \mathrm{~h}$ at room temperature. Membranes were incubated overnight at $4{ }^{\circ} \mathrm{C}$ with the primary antibody (phosphorERK $1 / 2$ at 1:2000, total-ERK at 1:1000, EGFR at 1:1500, rabbit anti-human HER-2 at 1:1000 [Cell Signaling Technology, MA, USA], and mouse anti-human beta-actin at 1:3000 [Sigma-Aldrich]). After incubation, the membranes were washed 5 times with TBST and incubated with the appropriate secondary antibody conjugated to horseradish peroxidase (anti-rabbit or mouse at 1:40000, BIO-RAD) for $1 \mathrm{~h}$ at room temperature. After washing 5 more times with TBST, the membranes were incubated with Immobilon Western Detection reagent (Millipore, Billerica, MA, USA) for $5 \mathrm{~min}$ and detected by an Image Quant Imager (GE Healthcare Bio Science). The expression levels of phosphorylated ERK were quantified by scanning the digital image and digitized data were analyzed with the Image J (NIH, USA).

\section{RNA isolation and quantitative RT-PCR}

RNA was extracted from the endometrial cancer cell lines and primary resected endometrioid adenocarcinoma tissue samples. Total cellular and tissue RNA were extracted using Isogen (WAKO, Osaka, Japan) and $2 \mu \mathrm{g}$ total RNA was treated with DNase I (Isogen, De Meern, Netherlands) according to manufacturer's protocol. RNA was reverse transcribed using SuperScript III transcriptase (Invitrogen) with random primers (Invitrogen). The samples were incubated with RNAse at $37{ }^{\circ} \mathrm{C}$ to remove RNA, and were diluted with distilled water for a final volume of $100 \mu \mathrm{L}$. Each quantitative PCR consisted of $5 \mu \mathrm{L}$ of cDNA template, $12.5 \mu \mathrm{L}$ SYBR Green real-time PCR master mix (Toyobo, Osaka, Japan), $0.2 \mu \mathrm{L}$ forward and reverse primers $(50 \mu \mathrm{M})$, and $7.1 \mu \mathrm{L}$ distilled water. The sequences for the forward and reverse primers are as follows: human EGFR: 5' -GGAGAACTGCCAGAAACTGACC- 3' and 5' -GCCTGCAGCACACTGGTTG- 3'; human HER-2: 5' -ATCTGGCGCTTTTGGCACAG- 3' and 5' -CACCA GCCATCACGTATGCT- 3'; human GAPDH: 5' -AATTC CATGGCACCGTCAAG- 3' and 5' -GGTGAAGACGCC AGTGGACT- 3'. The reactions were carried out in an ABI PRISM 7000 sequence detection system (Applied Biosystems, Foster City, CA, USA) for 40 cycles $\left(95^{\circ} \mathrm{C}\right.$ for $15 \mathrm{~s}$, $60{ }^{\circ} \mathrm{C}$ for $1 \mathrm{~min}$ ) after $1 \mathrm{~min}$ initial incubation at $95{ }^{\circ} \mathrm{C}$. The fold change in the expression levels of each gene was calculated using the standard curve method, with GAPDH as an internal control.

\section{siRNA transfection}

SiRNA against human EGFR (EGFR siRNA) or HER-2 (HER-2 siRNA), and siRNA for negative control (control siRNA) were obtained from Applied Biosystems. All cell lines were plated for $24 \mathrm{~h}$ to approximately $50 \%$ confluence, and were transfected with $10 \mathrm{nM}$ siRNA using Lipofectamine RNAiMAX (Ambion, Grand Island, NY, USA). The transfected cells were subjected to western 
Table 1 Characteristics of surgical cancer patients

\begin{tabular}{llll}
\hline & $\begin{array}{l}\text { Well differentiated } \\
(\mathrm{G} 1)(n=4)\end{array}$ & $\begin{array}{l}\text { Mediate differentiated } \\
(\mathrm{G} 2)(n=17)\end{array}$ & $\begin{array}{l}\text { Poorly differentiated } \\
(\mathrm{G} 3)(n=10)\end{array}$ \\
\hline $\mathrm{Age}^{\dagger}$ & $57(50-63)$ & $59(56-66)$ & $58(52-61)$ \\
$\mathrm{BMI}^{\dagger}$ & $22.2(20.0-28.6)$ & $23.4(20.3-25.0)$ & $21.4(20.7-23.6)$ \\
FIGO Surgical Stage & & & $5(50.0 \%)$ \\
I & $16(66.7 \%)$ & $10(58.8 \%)$ & $0(0.0 \%)$ \\
II & $3(12.5 \%)$ & $1(5.9 \%)$ & $3(30.0 \%)$ \\
III & $4(16.7 \%)$ & $6(35.3 \%)$ & $2(20.0 \%)$ \\
IV & $1(4.2 \%)$ & $0(0 \%)$ & $\mathrm{ns}$ \\
\hline
\end{tabular}

${ }^{\dagger}$ Median (Interquartile Range). FIGO International Federation of Gynecology and Obstetrics

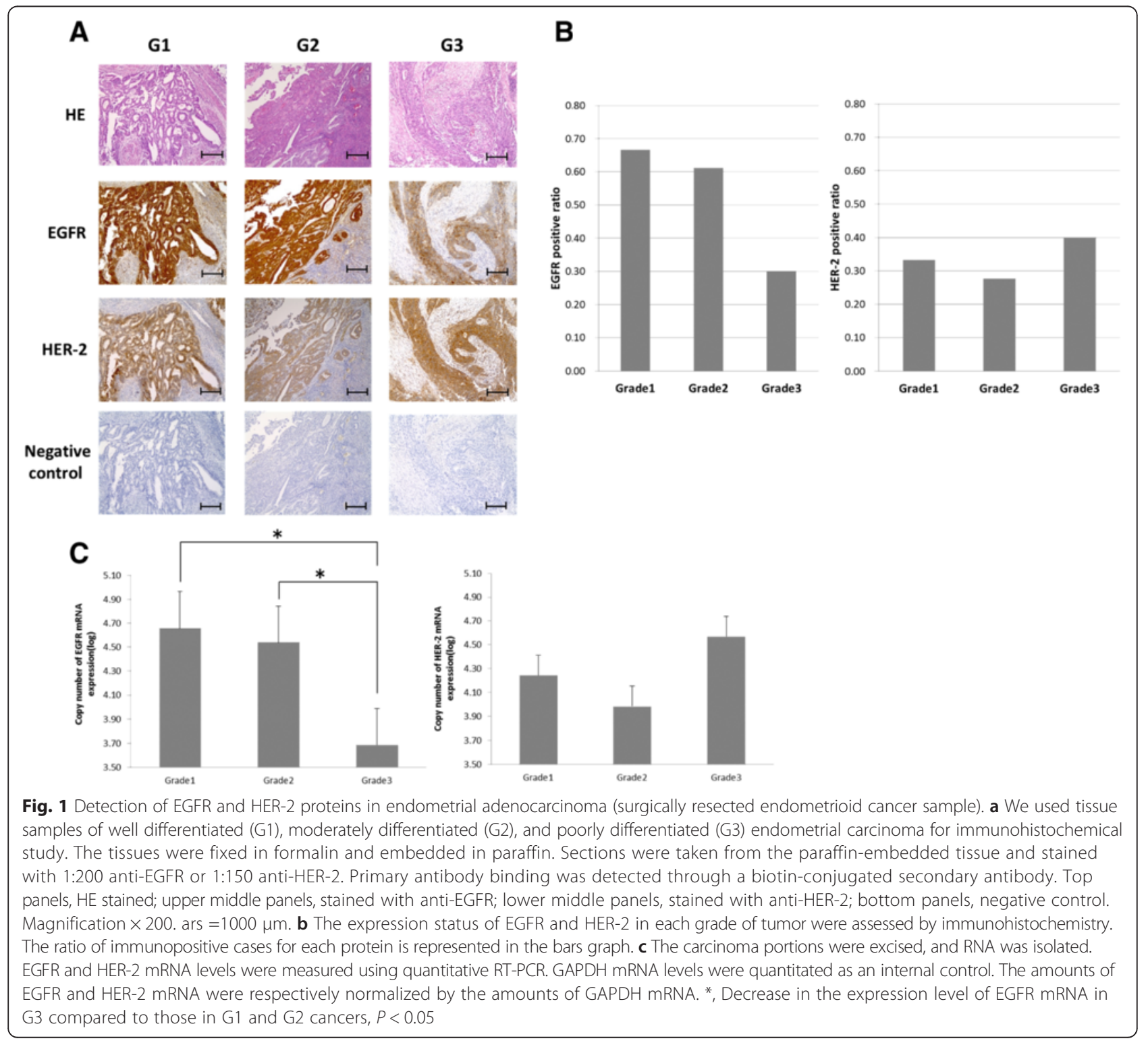


blotting, quantitative RT-PCR, and growth inhibition assay.

\section{Growth inhibition assay}

Cells were plated at a concentrations of 5000 cells (Ishikawa, KLE) or 10,000 cells (HEC-1A) per well in 96-well plates. After $12 \mathrm{~h}$ incubation at $37^{\circ} \mathrm{C}$ in a humidified atmosphere containing $5 \% \mathrm{CO}_{2}$, the cells were treated with the pharmacological compounds (ErbB inhibitor: erlotinib [EGFR tyrosine kinase inhibitor) and trastuzumab [HER-2 monoclonal antibody]) or transfected with siRNA, and incubated for further $48 \mathrm{~h}$ in the same conditions. Erlotinib was dissolved in DMSO and added to the cell culture medium at a concentration not exceeding $0.1 \%(\mathrm{v} / \mathrm{v})$. At the end of various treatments, $10 \mu \mathrm{L}$ cell counting solution (WST-1, Dojindo Labs, Tokyo, Japan) was added. Cells were incubated for $1 \mathrm{~h}$ and the absorbance was measured at a wavelength of 450-650 nm using a Microtiter Plate Reader (Becton Dickinson, Franklin Lakes, NJ, USA).

\section{Tumor xenograft model and treatment}

All procedures involving animals followed ethical principles according the NIH Guide for Care and Use of the Laboratory Animals and were approved by the Gunma University Animal Care and Use Committee (Permit Number: 13-042). Female mice, 4-weeks-old nude BALB/ $\mathrm{C} \mathrm{nu/nu}$, were obtained from Charles River Japan (Tokyo, Japan). Mice were housed in suitable cages in a pathogenfree condition in a room maintained at $23-26^{\circ} \mathrm{C}, 50 \% \mathrm{hu}$ midity, and 12-h light/12-h dark cycle. The mice were allowed to acclimatize for 2 weeks prior to the study. Regular health checks were performed. Mice were implanted with tumor cells at a single subcutaneous (s.c.) site on the shoulder flank $\left(5 \times 10^{5}\right.$ HEC- 1 and $1 \times 10^{6}$ Ishikawa per mouse in a $0.1 \mathrm{~mL}$ growth factor reduced matrigel (Corning, Tewksbury, MA, USA) and $0.1 \mathrm{~mL}$ culture medium. Tumor-bearing mice were randomized into erlotinib $(1 \mathrm{mg}$, $3 \mathrm{mg}, 10 \mathrm{mg}, 30 \mathrm{mg} / \mathrm{kg} /$ day, intraperitoneal (i.p.) for 5 days per week), pertuzumab ( $1 \mathrm{mg}, 3 \mathrm{mg}, 10 \mathrm{mg} / \mathrm{kg}$, i.p. twice per week), and vehicle (DMSO and distilled water, i.p.)

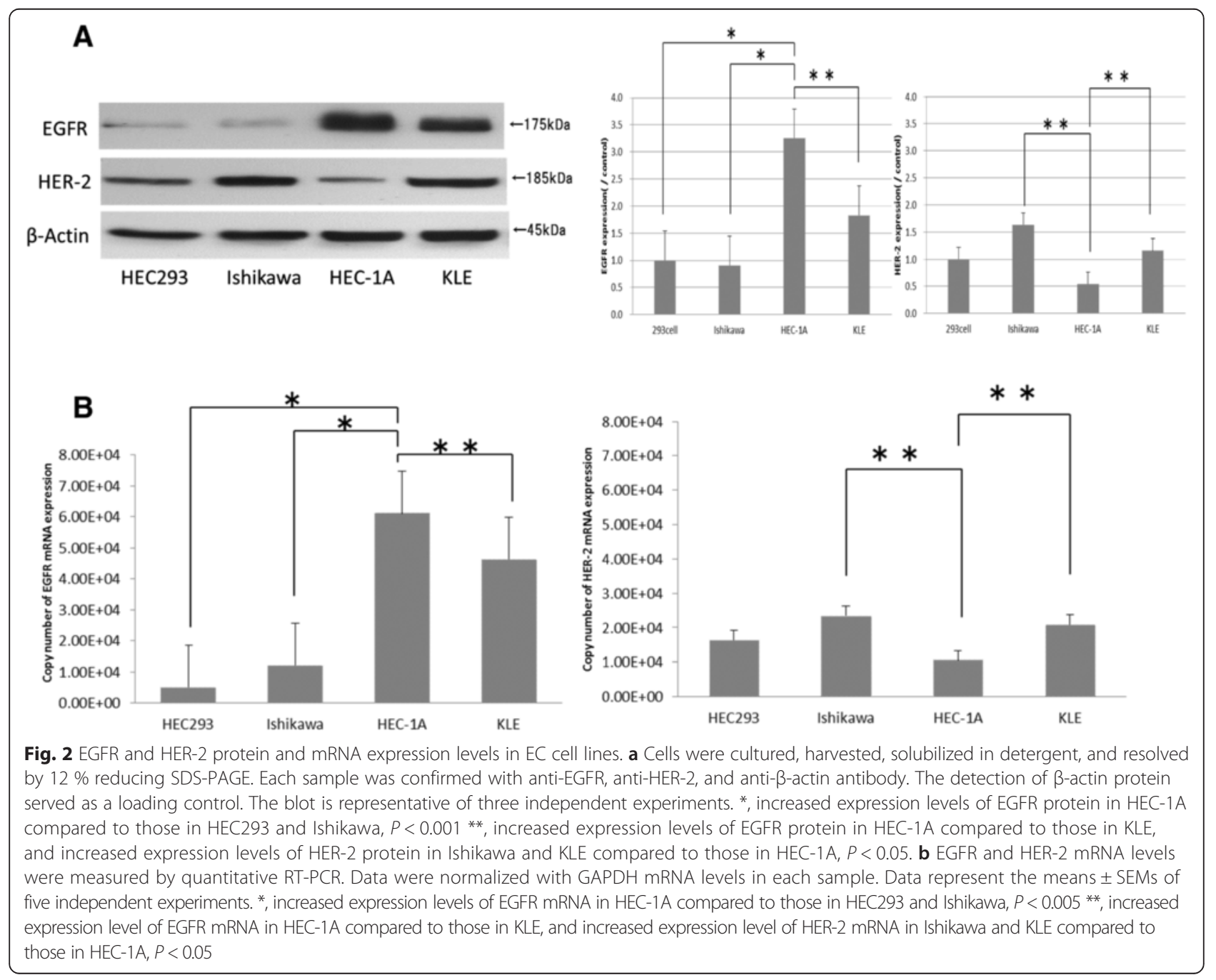


groups when the mean tumor volume was $100-150 \mathrm{~mm}^{3}$. Equal volume of the vehicle $(0.1 \mathrm{~mL})$ was injected in all animals. Tumor volume and body weight were determined twice weekly. The tumor volume was determined according to the following formula: tumor volume $=($ length $) \mathrm{x}$ (width) $)^{2} / 2$. On day 28 , mice were euthanized; tumor was excised, and fixed in formalin. Tumors were processed for hematoxylin and eosin (HE) staining.

\section{Data analysis}

The data represent the mean \pm standard error of the mean (SEM) from at least three independent experiments.

Comparisons between groups were performed by oneway ANOVA or chi-square test. The significance of the differences between the mean values of the control group and each treated group was determined by Dunnett's multiple-comparison test. These analyses were 2-tailed tests, and a value of $P<0.05$ was considered significant. The cumulative survival curve was estimated by the Kaplan-Meier method. All analyses were performed with IBM SPSS statistics 21 software.

\section{Results}

Expression of EGFR and HER-2 in endometrial cancer

Fifty-one surgically resected endometrioid carcinoma samples, classified as: well (Grade 1, G1), moderately (G2), or poorly (G3) differentiated adenocarcinoma, were obtained from patients who had undergone surgery at Gunma University Hospital (Table 1). In our institution, $20.9 \%$ of patients with endometrial cancer with low-grade endometrioid histology were diagnosed as stage III and IV. As a first step, IHC was carried out on endometrial carcinoma to confirm the expression of EGFR and HER-2 proteins (Fig. 1a and b). EGFR protein was highly expressed in G1 and G2 endometrioid carcinoma ( $p=0.014, \chi^{2}$ $(2)=8.6)$ whereas HER-2 was almost evenly expressed in G1, G2, and G3 tumors $\left(P=0.52, X^{2}(2)=1.5\right)$. We also evaluated EGFR and HER-2 mRNA expression levels in EC tissues by RT-PCR (Fig. 1c). EGFR mRNA levels were higher in $\mathrm{G} 1$ and $\mathrm{G} 2(P<0.05)$ than in $\mathrm{G} 3$, but there was no significant difference in HER-2 mRNA expression between the three grades.

We did further Kaplan-Meier analysis of survival with patients who expressed or not EGFR by IHC. No significant differences were found between the two groups for overall survival (data not shown).

\section{EC cell line experiments}

Cancer cell lines were utilized for further experiments to elucidate the roles of EGFR and HER-2 in EC cells. Three cell lines (Ishikawa, HEC-1A, and KLE) were evaluated by western blotting to determine protein expression

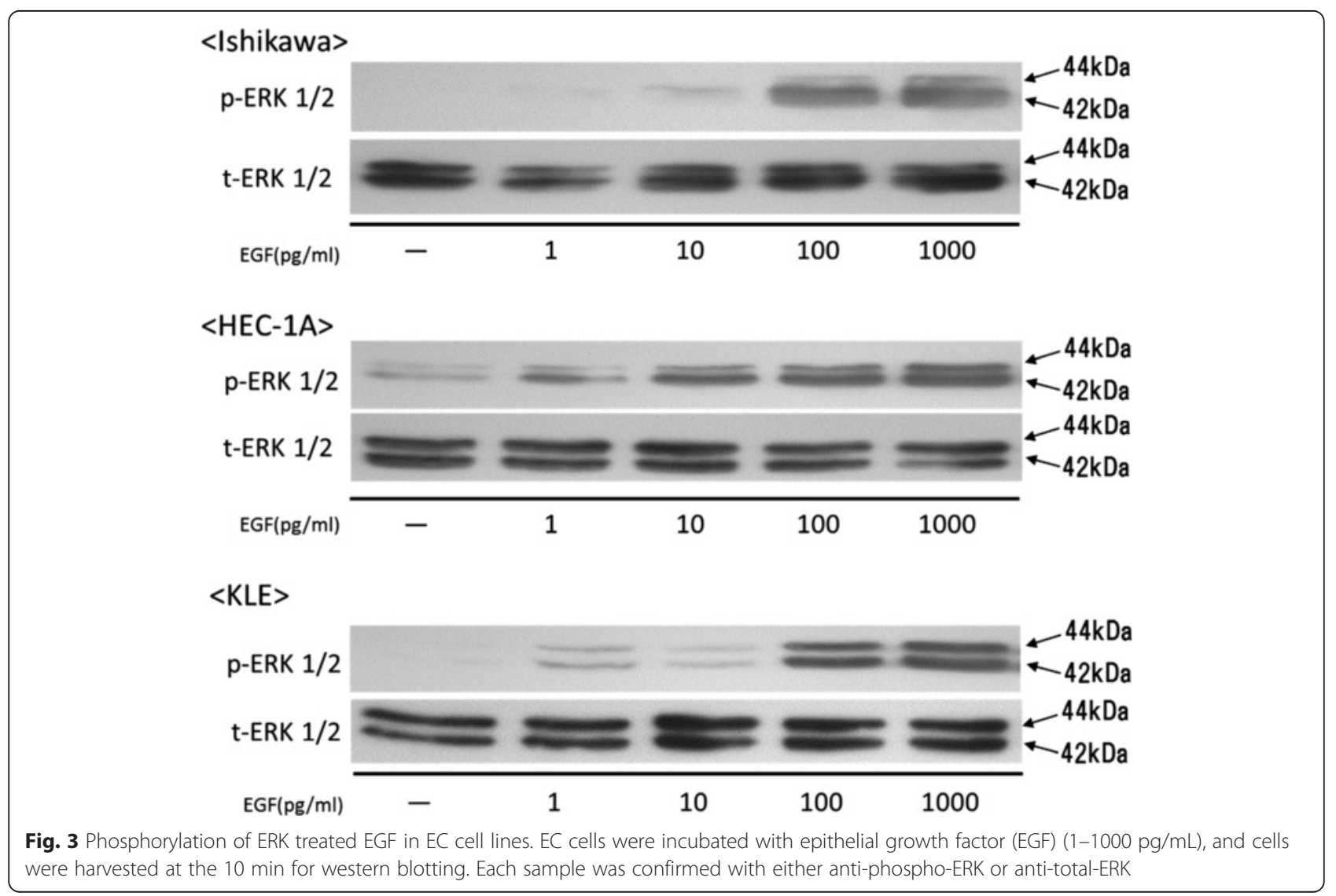


levels of EGFR and HER-2. HEC-1A showed high EGFR and low HER-2 expression, while Ishikawa had low EGFR and high HER-2 expression. In KLE, the expression levels of EGFR and HER-2 were intermediate between Ishikawa and HEC-1A (Fig. 2a). These results were reconfirmed by quantitative RT-PCR experiments, which indicated that EGFR mRNA levels were significantly the highest in HEC1A $(P<0.005)$, and HER-2 mRNA levels were highly expressed in Ishikawa $(P<0.05)$ (Fig. 2b).

The three cell lines were treated with EGF and were evaluated for downstream signaling of EGFR, by detecting phosphorylated ERK $1 / 2$ by western blotting (Fig. 3). The phosphorylation of ERK $1 / 2$ was found to be induced in all three cell lines, but in HEC-1A, the increase occurred at a lower concentration compared to the other two cell lines. This result suggested that the amount of EGFR expression was an important factor for the activation of mitotic-activated protein kinase (MAPK) pathway by EGF stimulation in endometrial carcinoma cells.

To investigate the significance of EGFR and HER-2 in the proliferation of endometrial cancer cells, all cells were transfected with siRNA to knock down EGFR or HER-2. After 48 h, EGF was added, and ERK 1/2 phosphorylation and proliferation were evaluated. When EGFR was knocked down (Fig. 4a and c), all cells showed decreased ERK $1 / 2$ phosphorylation $(P<0.05)$. The viability of Ishikawa cells was reduced to $72 \%$, HEC- $1 \mathrm{~A}$ to $57 \%$, and KLE to $64 \%$, compared to the negative control $(P<0.05)$. When HER-2 was knocked down (Fig. $4 \mathrm{~b}$ and c), ERK $1 / 2$ phosphorylation was significantly decreased in Ishikawa, which highly expressed HER-2 $(P<0.05)$, but not in HEC$1 \mathrm{~A}$ and KLE. Similarly, cell viability was reduced in Ishikawa (to $65 \%$ compared with negative control) $(P<0.05)$, but not in other cell types (HEC-1A: to $94 \%$ KLE: to $93 \%$ compared with negative control).

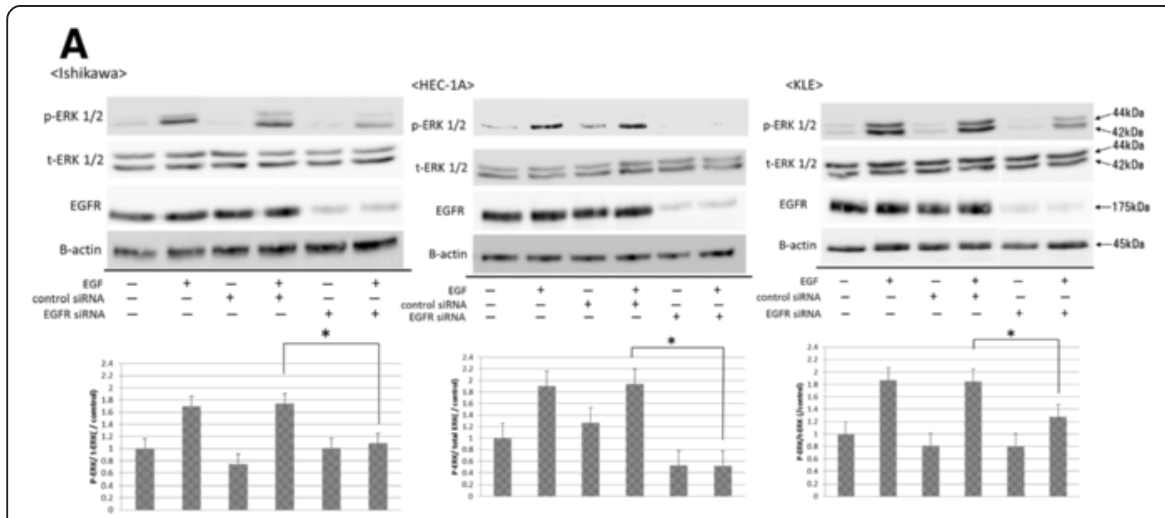

\section{B}

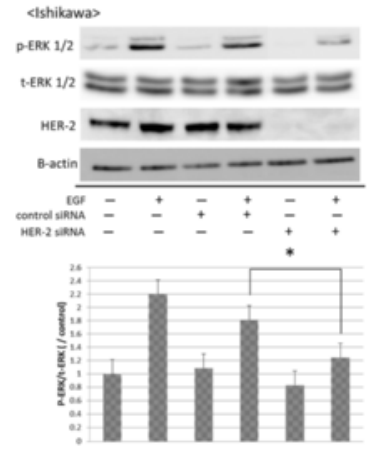

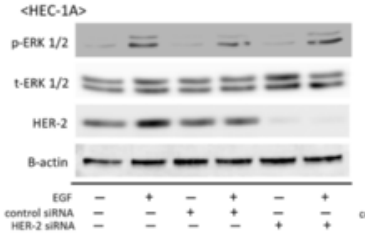

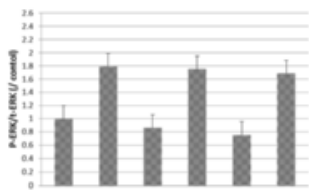

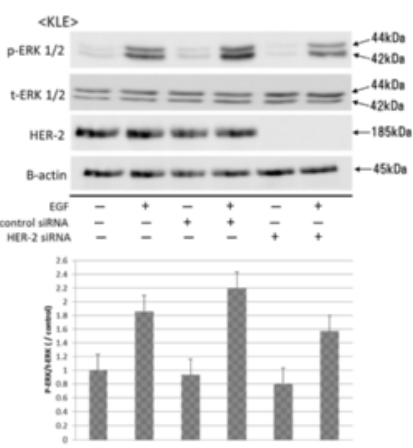
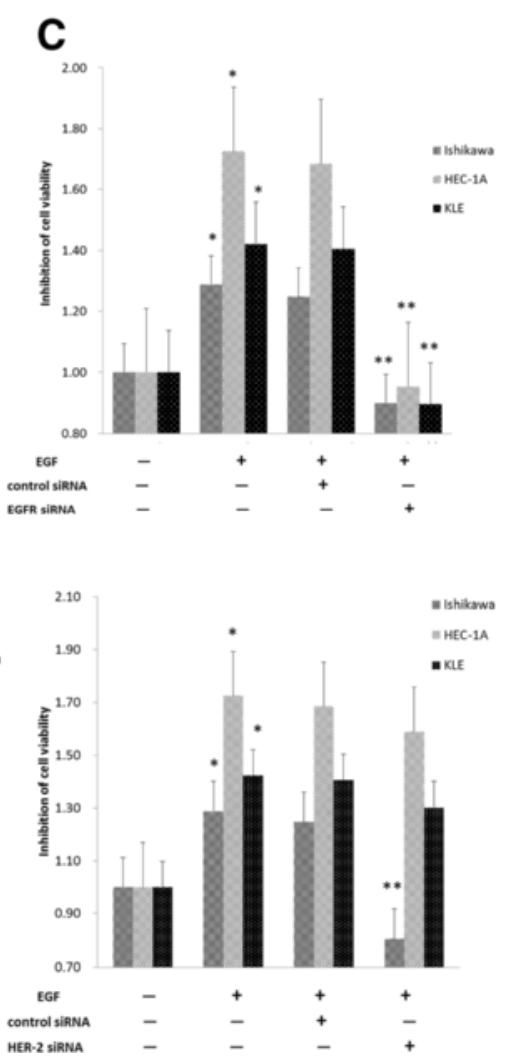

Fig. 4 EGFR is involved in ERK phosphorylation in EC cell lines. All EC cells were transfected with $10 \mathrm{nM}$ of siRNA (control, EGFR, or HER-2). Cells were harvested $48 \mathrm{~h}$ after transfection to evaluate ERK phosphorylation after knockdown of EGFR (a) or HER-2 protein (b). Cells were incubated with EGF $(1 \mathrm{ng} / \mathrm{mL})$ for $10 \mathrm{~min}$ and harvested for western blot analysis. The detection of $\beta$-actin protein served as a loading control. The blot is representative of three independent experiments. The expression levels of phosphorylated ERK were quantified by scanning the digital image and digitized data were analyzed with the Image J. Data represent the means \pm SEMs of three independent experiments. * decreased compared to siRNA control transfection (NC), $P<0.05$. c All EC cells were transfected with $10 \mathrm{nM}$ of siRNA (control, EGFR or HER-2), and cell proliferation was monitored after $48 \mathrm{~h}$ using WST-1 assay. ${ }^{*}$, increased compared to no treatment cell, $P<0.05{ }^{* *}$, decreased compared to siRNA control transfection (Negative Control), $P<0.05$ 


\section{Growth inhibition assay following ErbB inhibitor treatment in vitro}

The results in Fig. 4 prompted us to investigate whether ErbB inhibitors could effectively inhibit EC proliferation. In subsequent experiments, all cells were treated with erlotinib (ERL: EGFR tyrosine kinase inhibitor) or trastuzumab (TRA: HER-2 monoclonal antibody), and evaluated for ERK $1 / 2$ phosphorylation and proliferation in EC cells. All cells treated with ERL showed decreased ERK $1 / 2$ phosphorylation $(P<0.001)$ (Fig. 5a) and reduction in cell viability. However, ERL had a most pronounced effect to HEC-1A compared with Ishikawa and KLE in cell viability (HEC-1A $38 \%$, Ishikawa $78 \%$, and KLE $72 \%$, respectively) (Fig. 5b). In the case of TRA treatment (Fig. 5a and b), only Ishikawa cells showed a decrease in ERK $1 / 2$ phosphorylation $(P<0.05)$ and cell viability to $78 \%$ compared with vehicle control $(P<0.05)$.

\section{Tumor growth inhibition assay following ErbB inhibitor treatment in mice xenograft model}

Because the in vitro studies were examined for short periods, the long-term effect of either ERL or TRA was studied using an EC xenograft in vivo model. Tumor-bearing mice were treated with either ERL or TRA for 28 days. The results showed that only tumors in HEC-1A xenografted mice treated with ERL at a dose of $3 \mathrm{mg} / \mathrm{kg}$ or more (Fig. $6 \mathrm{a}$ and b) were reduced, whereas TRA did not induce significant tumor growth inhibition in mice implanted with either HEC-1A or Ishikawa. The resected tumor from the xenograft model stained with $\mathrm{HE}$, suggested that clear fibrosis occurred in HEC-1A tumor treated with ERL (Fig. 6c).

\section{Discussion}

In the present study, we demonstrate that both EGFR mRNA and EGFR protein were highly expressed in low-grade endometrioid carcinoma, but the expression

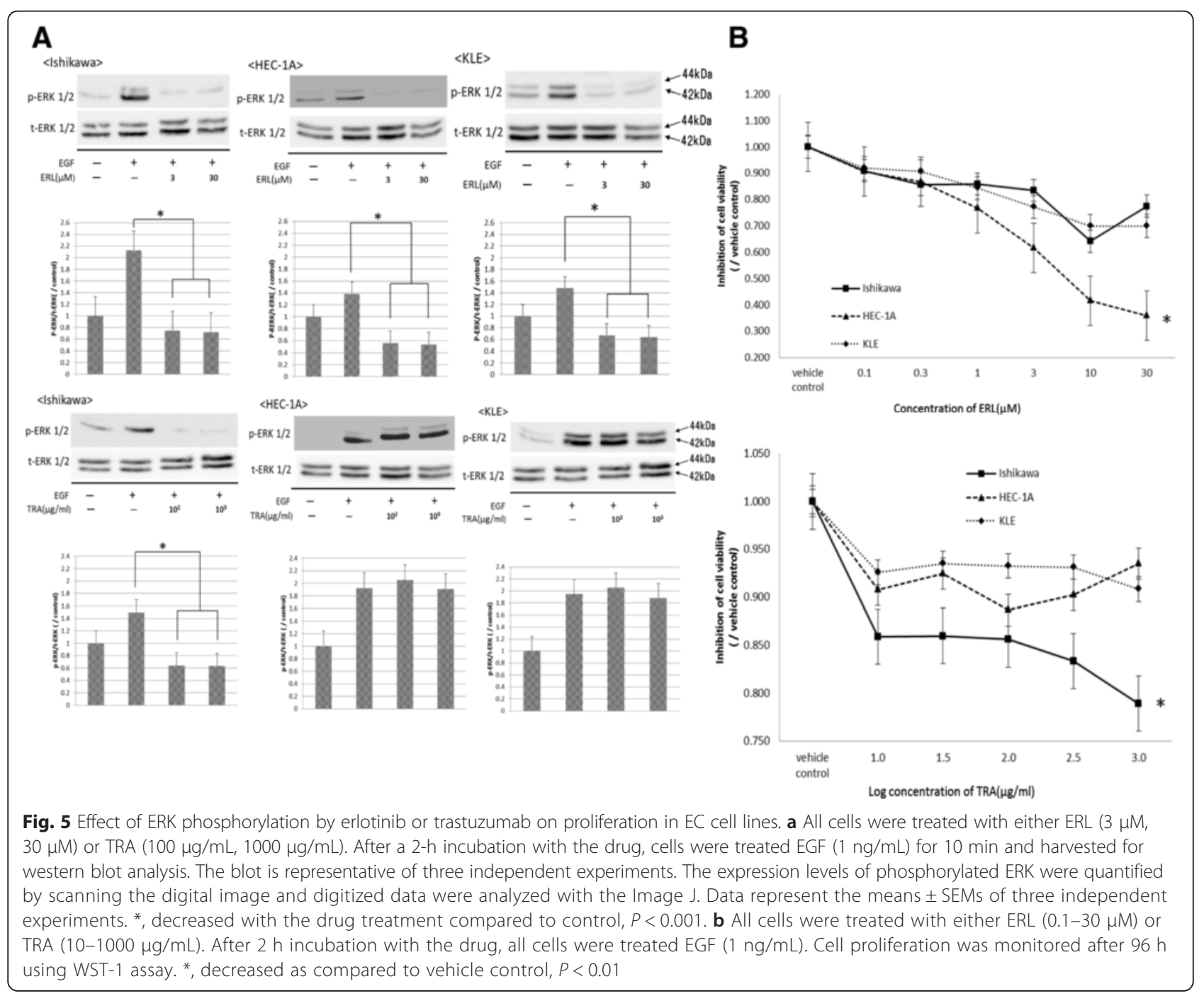




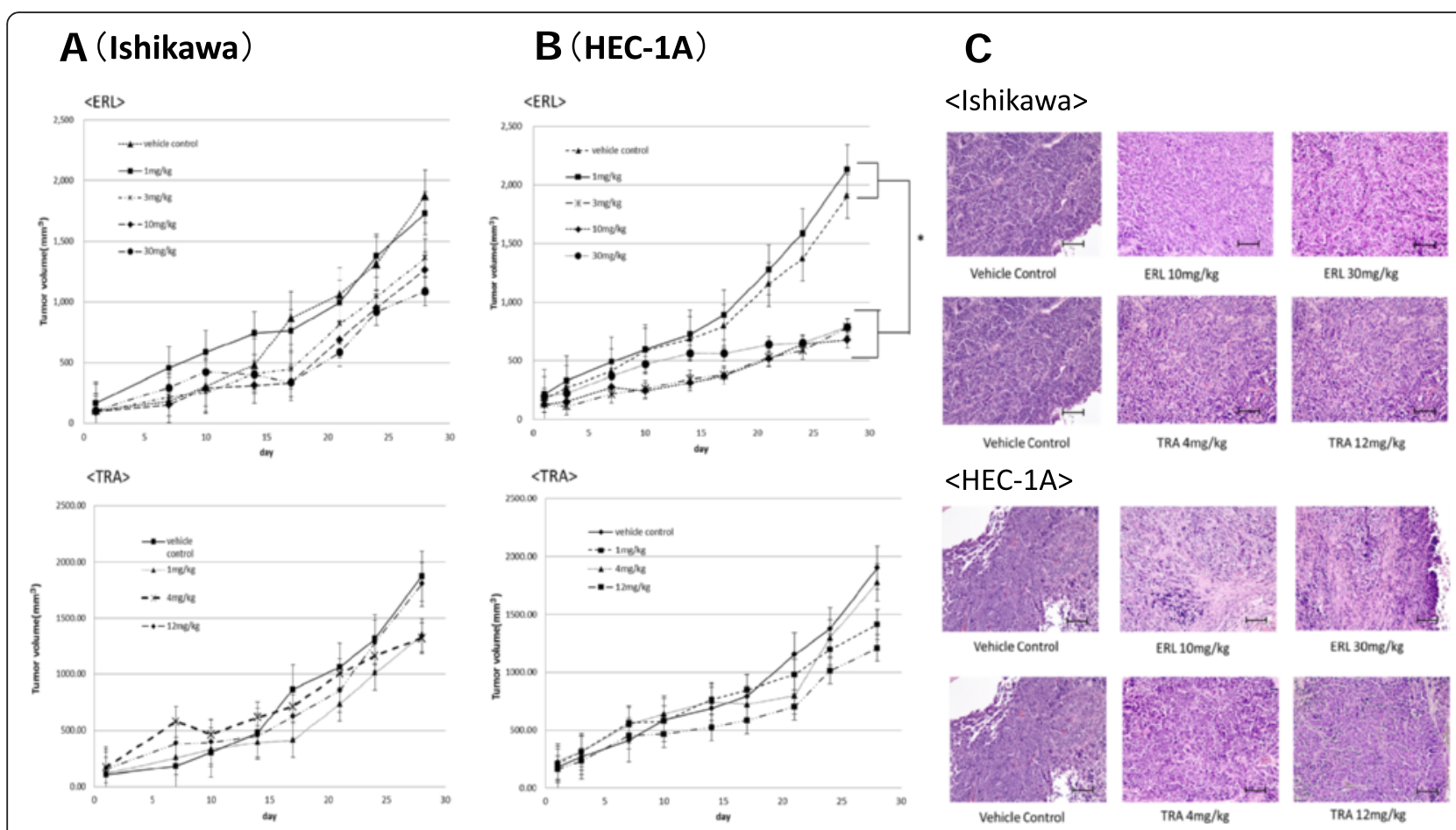

Fig. 6 Inhibition of tumor growth by erlotinib (ERL) in vivo. Mice were implanted with Ishikawa (a) or HEC-1A (b) and treated with ERL or TRA for 28 days. Tumor volume was measured twice a week. Data represent the means \pm SEMs of three independent tumor volumes. ${ }^{*}$, decreased as compared to vehicle control, $P<0.05$. c On day 28 after starting treatment, mice were euthanized and tumor was excised. The tissues were fixed in formalin and embedded in paraffin. Sections were taken from the paraffin-embedded tissue and HE stained. Top and upper middle panels, Ishikawa tumor; lower middle and bottom panels, HEC-1A tumor. Magnification $\times 200$.Bars $=1000 \mu \mathrm{m}$

was low in high-grade endometrioid carcinoma. We examined the molecular factors that underlie the variable responsiveness to erlotinib in accordance with the expression levels of both EGFR mRNA and EGFR protein in the endometrial carcinoma cells, using quantitative RT-PCR and IHC. We found that erlotinib, a known potent selective inhibitor of the EGFR tyrosine kinase, significantly inhibits the proliferation of endometrial carcinoma cells, which express high levels of EGFR in xenograft mice models.

The degree of tumor differentiation is one of the prognostic factors in EC; low-grade endometrioid tumors tend not to progress to deep myometrial invasion or spread to distant sites [35]. In contrast, high-grade endometrioid tumor is aggressive and diagnosed at advanced stages, it involves recurrent or metastatic tumors at high rate. On the other hand, overall prognosis for those who are diagnosed with low-grade tumor is positive, although the number of patients with recurrent or metastatic tumors is still large due to the corresponding amount of newly diagnosed type I EC patients [3, 4]. In fact, in our institution, $20.9 \%$ of endometrial cancer patients with low-grade endometrioid histology have been diagnosed as stage III and IV (Table 1). We comprehensively analyzed EGFR and HER2 expression levels in endometrioid carcinoma
(Fig. 1), demonstrating that EGFR mRNA and protein were highly expressed in low-grade endometrioid tumor compared to high-grade endometrioid tumor. In contrast, HER2 was not significantly expressed at a varying level in any grade of endometrioid tumor. EGFR mediates the activation of intracellular signaling pathways, such as MAPK-ERK and PI3K-AKT pathways, resulting in enhanced proliferation and cell survival in EC cells. Collectively, these results prompted us to further investigate the significance of EGFR in the proliferation of low-grade endometrioid tumor.

To date, anti-EGFR antibody, anti-EGFR, or dual EGFR/ HER2 tyrosine kinase inhibitors have been evaluated across a variety of disease types. For HER2-positive patients with breast cancer, trastuzumab has significantly reduced the rate of recurrence [36]. In the subsequent study [37], lapatinib, the EGFR and HER2 dual kinase inhibitor, displayed significant antiproliferative effects in HER2 overexpressing breast tumor cell lines, suggesting that the EGFR expression level has no association with the sensitivity to lapatinib. In contrast, both EGFR and HER2 expression has been found in patients with non-small-cell lung cancer with poor prognosis [38], erlotinib was beneficial in those patients in an EGFR-dependent way [39]. In this study, we confirmed that the amount of EGFR expression plays an important 
role in MAPK pathway in endometrial cancer cell (Fig. 3), suggesting that endometrial cancer with highly expressed EGFR is strongly effected by anti-EGFR drugs. Furthermore, the antitumor effects of erlotinib against HEC-1A cells clearly inhibited tumor growth both in vitro (Fig. 5b) and in vivo (Fig. 6b). On the other hand, trastuzumab did not reduce the tumor growth of Ishikawa cells in xenograft mice (Fig. 6a). Taken together, the current data indicate that the expression levels of EGFR is a key factor in the molecular targeted therapy against pathogenic tyrosine kinases in endometrial cancer, and suggest that EGFR inhibitors may be clinically useful for well-defined subgroups of endometrial cancer patients with highly-expressed EGFR.

A phase II study (NCIC IND-148) has been largely considered to have concluded that erlotinib is not a promising agent for recurrent or metastatic EC. However, in that study, tumors were regarded as EGFR positive if tumor cell membranes stained positively with anti-EGFR antibody in IHC in more than $10 \%$ of tumor cells. Thus, we speculate that this clinical study contained large cases of high-grade endometrioid tumors and type II EC, based on our finding that a majority of cell membranes were stained in low-grade endometrioid tumors (Fig. 1).

Patients with risk factors such as tumor grade, deep myometrial invasion, and positive lymph nodes are recommended for systemic chemotherapy, although it is not unanimously accepted. Basic cancer research is conducted to identify the markers that determine patients to chemotherapy regimen according to the responses. In malignant tumors, it is unlikely that one signaling pathway is solely engaged in its aggressive behavior including progression and metastasis. However, the present data demonstrate that erlotinib has efficacy in the treatment of endometrial cancers, which highly express EGFR. We believe that further analysis of the molecular signature of the EC tumors will define patients who can be benefited by erlotinib therapy.

\section{Conclusions}

Type I EC accounting for $80 \%$ of EC is associated with better clinical outcome than type II EC. However, a substantial number of patients have to deal with recurrent tumors due to corresponding amounts of type I EC patients. In this study, we found that EGFR protein was highly expressed in low grade endometrioid carcinoma tissue and that the proliferation of an EC cell line with high EGFR expression was attenuated by erlotinib, an EGFR tyrosine kinase inhibitor. In tumor xenograft mouse models, erlotinib clearly reduced the growth of tumors with high levels of EGFR expression. This agent could be further developed for pre-clinical and clinical studies specific patient subgroups in advanced stage or recurrent EC with high-EGFR expressed tumors.

\section{Abbreviations}

EC: Endometrial carcinoma; EGF: Epidermal growth factor; EGFR: Epidermal growth factor receptor; HER-2: Human epidermal growth factor receptor type2; ERK1/2: Extracellular Signal-regulated Kinase 1/2; MAPK: Mitotic-activated protein kinase; ERL: Erlotinib; TRA: Trastuzumab; IHC: Immunohistochemistry.

\section{Competing interests}

The authors declare that they have no competing interests.

\section{Authors' contributions}

TN performed the in vitro experiments. TN and KN planned and designed the experiments. SY, SI, KK, and TN performed the xenograft model experiments. TN and KN analyzed and interpreted the data. TM participated in its design and coordination. KN helped to draft the manuscript. All authors read and approved the final manuscript.

\section{Acknowledgements}

We thank Hiroko Matsuda and Junko Sakurai for their excellent technical assistance and the Departments of Diagnostic Pathology in Gunma University Graduate School of Medicine for the in vivo technical support.

\section{Author details}

'Department of Obstetrics and Gynecology, Gunma University, 3-39-22, Showa, Maebashi, Gunma 371-8511, Japan. ${ }^{2}$ Gunma Prefectural Cancer Center, 617-1, Nishimachi, Takabayashi, Ota, Gunma 373-8500, Japan.

Received: 12 August 2015 Accepted: 5 December 2015

Published online: 16 December 2015

\section{References}

1. Bokhman JV. Two pathogenetic types of endometrial carcinoma. Gynecol Oncol. 1983;15(1):10-7.

2. Deligdisch L, Holinka CF. Endometrial carcinoma: two diseases? Cancer Detect Prev. 1987:10(3-4):237-46.

3. Zagouri F, Bozas G, Kafantari E, Tsiatas M, Nikitas N, Dimopoulos MA, et al. Endometrial cancer: what is new in adjuvant and molecularly targeted therapy? Obstet Gynecol Int. 2010;749579.

4. Aoki D. Annual report of Gynecologic Oncology Committee, Japan Society of Obstetrics and Gynecology. J Obstet Gynaecol Res. 2013;40(2):338-48.

5. Creasman WT, Odicino F, Maisonneuve P, Quinn MA, Beller U, Benedet JL, et al. Carcinoma of the corpus uteri. FIGO 26th Annual Report on the Results of Treatment in Gynecological Cancer. Int J Gynaecol Obstet. 2006;95 Suppl 1:S105-43.

6. Creutzberg CL, van Putten WL, Koper PC, Lybeert ML, Jobsen JJ, WarlamRodenhuis CC, et al. Surgery and postoperative radiotherapy versus surgery alone for patients with stage-1 endometrial carcinoma: multicentre randomised trial. PORTEC Study Group. Post Operative Radiation Therapy in Endometrial Carcinoma. Lancet. 2000;355(9213):1404-11.

7. Keys HM, Roberts JA, Brunetto VL, Zaino RJ, Spirtos NM, Bloss JD, et al. A phase III trial of surgery with or without adjunctive external pelvic radiation therapy in intermediate risk endometrial adenocarcinoma: a Gynecologic Oncology Group study. Gynecol Oncol. 2004;92(3):744-51.

8. Randall ME, Filiaci VL, Muss H, Spirtos NM, Mannel RS, Fowler J, et al. Randomized phase III trial of whole-abdominal irradiation versus doxorubicin and cisplatin chemotherapy in advanced endometrial carcinoma: a Gynecologic Oncology Group Study. J Clin Oncol. 2006;24(1):36-44.

9. Kuoppala T, Maenpaa J, Tomas E, Puistola U, Salmi T, Grenman S, et al. Surgically staged high-risk endometrial cancer: randomized study of adjuvant radiotherapy alone vs. sequential chemo-radiotherapy. Gynecol Oncol. 2008;110(2):190-5.

10. Thigpen JT, Blessing JA, DiSaia PJ, Yordan E, Carson LF, Evers C. A randomized comparison of doxorubicin alone versus doxorubicin plus cyclophosphamide in the management of advanced or recurrent endometrial carcinoma: A Gynecologic Oncology Group study. J Clin Oncol. 1994;12(7):1408-14.

11. Thigpen JT, Brady MF, Homesley HD, Malfetano J, DuBeshter B, Burger RA, et al. Phase III trial of doxorubicin with or without cisplatin in advanced endometrial carcinoma: a gynecologic oncology group study. J Clin Oncol. 2004;22(19):3902-8.

12. van Wijk FH, Aapro MS, Bolis G, Chevallier B, van der Burg ME, Poveda A, et al. Doxorubicin versus doxorubicin and cisplatin in endometrial 
carcinoma: definitive results of a randomised study (55872) by the EORTC Gynaecological Cancer Group. Ann Oncol. 2003;14(3):441-8.

13. Fleming GF, Filiaci VL, Bentley RC, Herzog T, Sorosky J, Vaccarello L, et al. Phase III randomized trial of doxorubicin + cisplatin versus doxorubicin + 24-h paclitaxel + filgrastim in endometrial carcinoma: a Gynecologic Oncology Group study. Ann Oncol. 2004;15(8):1173-8.

14. Fleming GF, Brunetto VL, Cella D, Look KY, Reid GC, Munkarah AR, et al. Phase III trial of doxorubicin plus cisplatin with or without paclitaxel plus filgrastim in advanced endometrial carcinoma: a Gynecologic Oncology Group Study. J Clin Oncol. 2004;22(11):2159-66.

15. Rusch V, Klimstra D, Venkatraman E, Pisters PW, Langenfeld J, Dmitrovsky E. Overexpression of the epidermal growth factor receptor and its ligand transforming growth factor alpha is frequent in resectable non-small cell lung cancer but does not predict tumor progression. Clin Cancer Res. 1997:3(4):515-22.

16. Brabender J, Danenberg KD, Metzger R, Schneider PM, Park J, Salonga D, et al. Epidermal growth factor receptor and HER2-neu mRNA expression in non-small cell lung cancer Is correlated with survival. Clin Cancer Res. 2001;7(7):1850-5.

17. Ekstrand AJ, James CD, Cavenee WK, Seliger B, Pettersson RF, Collins VP. Genes for epidermal growth factor receptor, transforming growth factor alpha, and epidermal growth factor and their expression in human gliomas in vivo. Cancer Res. 1991;51(8):2164-72.

18. Salomon DS, Brandt R, Ciardiello F, Normanno N. Epidermal growth factor-related peptides and their receptors in human malignancies. Crit Rev Oncol Hematol. 1995;19(3):183-232.

19. Chow NH, Liu HS, Lee El, Chang CJ, Chan SH, Cheng HL, et al. Significance of urinary epidermal growth factor and its receptor expression in human bladder cancer. Anticancer Res. 1997;17(2B):1293-6.

20. Ueda S, Ogata S, Tsuda H, Kawarabayashi N, Kimura M, Sugiura Y, et al. The correlation between cytoplasmic overexpression of epidermal growth factor receptor and tumor aggressiveness: poor prognosis in patients with pancreatic ductal adenocarcinoma. Pancreas. 2004;29(1):e1-8.

21. King CR, Kraus MH, Aaronson SA. Amplification of a novel v-erbB-related gene in a human mammary carcinoma. Science. 1985;229(4717):974-6.

22. Grandis JR, Sok JC. Signaling through the epidermal growth factor receptor during the development of malignancy. Pharmacol Ther. 2004;102(1):37-46.

23. Khalifa MA, Mannel RS, Haraway SD, Walker J, Min KW. Expression of EGFR, HER-2/neu, P53, and PCNA in endometrioid, serous papillary, and clear cell endometrial adenocarcinomas. Gynecol Oncol. 1994;53(1):84-92.

24. Scambia G, Benedetti Panici P, Ferrandina G, Battaglia F, Distefano M, D'Andrea $\mathrm{G}$, et al. Significance of epidermal growth factor receptor expression in primary human endometrial cancer. Int J Cancer. 1994;56(1):26-30.

25. Niikura $\mathrm{H}$, Sasano H, Matsunaga G, Watanabe K, Ito K, Sato S, et al. Prognostic value of epidermal growth factor receptor expression in endometrioid endometrial carcinoma. Hum Pathol. 1995;26(8):892-6.

26. Thoury A, Descatoire V, Kotelevets L, Kannengiesser C, Bertrand G, Theou-Anton $\mathrm{N}$, et al. Evidence for different expression profiles for c-Met, EGFR, PTEN and the mTOR pathway in low and high grade endometrial carcinomas in a cohort of consecutive women. Occurrence of PIK3CA and K-Ras mutations and microsatellite instability. Histol Histopathol. 2014;29(11):1455-66.

27. Ejskjaer K, Sorensen BS, Poulsen SS, Forman A, Nexo E, Mogensen O. Expression of the epidermal growth factor system in endometrioid endometrial cancer. Gynecol Oncol. 2007;104(1):158-67.

28. Mori N, Kyo S, Nakamura M, Hashimoto M, Maida Y, Mizumoto Y, et al, Expression of HER-2 affects patient survival and paclitaxel sensitivity in endometrial cancer. Br J Cancer. 2010;103(6):889-98.

29. Konecny GE, Venkatesan N, Yang G, Dering J, Ginther C, Finn R, et al. Activity of lapatinib a novel HER2 and EGFR dual kinase inhibitor in human endometrial cancer cells. Br J Cancer. 2008;98(6):1076-84.

30. Konecny GE, Santos L, Winterhoff B, Hatmal M, Keeney GL, Mariani A, et al. HER2 gene amplification and EGFR expression in a large cohort of surgically staged patients with nonendometrioid (type II) endometrial cancer. Br J Cancer. 2009;100(1):89-95.

31. Oza AM, Eisenhauer EA, Elit L, Cutz JC, Sakurada A, Tsao MS, et al. Phase II study of erlotinib in recurrent or metastatic endometrial cancer: $\mathrm{NCIC}$ IND-148. J Clin Oncol. 2008;26(26):4319-25.

32. Fleming GF, Sill MW, Darcy KM, McMeekin DS, Thigpen JT, Adler LM, et al. Phase II trial of trastuzumab in women with advanced or recurrent, HER2positive endometrial carcinoma: a Gynecologic Oncology Group study. Gynecol Oncol. 2010;116(1):15-20.
33. Pecorelli S. Revised FIGO staging for carcinoma of the vulva, cervix, and endometrium. Int J Gynaecol Obstet. 2009;105(2):103-4.

34. Amant F, Mirza MR, Creutzberg CL. Cancer of the corpus uteri. Int J Gynaecol Obstet. 2012:119 Suppl 2:S110-7.

35. Morrow CP, Bundy BN, Kurman RJ, Creasman WT, Heller P, Homesley HD, et al. Relationship between surgical-pathological risk factors and outcome in clinical stage I and II carcinoma of the endometrium: a Gynecologic Oncology Group study. Gynecol Oncol. 1991;40(1):55-65.

36. Romond EH, Perez EA, Bryant J, Suman VJ, Geyer Jr CE, Davidson NE, et al. Trastuzumab plus adjuvant chemotherapy for operable HER2-positive breast cancer. N Engl J Med. 2005;353(16):1673-84.

37. Konecny GE, Pegram MD, Venkatesan N, Finn R, Yang G, Rahmeh M, et al. Activity of the dual kinase inhibitor lapatinib (GW572016) against HER-2overexpressing and trastuzumab-treated breast cancer cells. Cancer Res. 2006;66(3):1630-9.

38. Tateishi M, Ishida T, Kohdono S, Hamatake M, Fukuyama Y, Sugimachi K. Prognostic influence of the co-expression of epidermal growth factor receptor and c-erbB-2 protein in human lung adenocarcinoma. Surg Oncol. 1994:3(2):109-13.

39. Shepherd FA, Rodrigues Pereira J, Ciuleanu T, Tan EH, Hirsh V, Thongprasert $\mathrm{S}$, et al. Erlotinib in previously treated non-small-cell lung cancer. N Engl J Med. 2005:353(2):123-32

\section{Submit your next manuscript to BioMed Central and we will help you at every step:}

- We accept pre-submission inquiries

- Our selector tool helps you to find the most relevant journal

- We provide round the clock customer support

- Convenient online submission

- Thorough peer review

- Inclusion in PubMed and all major indexing services

- Maximum visibility for your research

Submit your manuscript at www.biomedcentral.com/submit
(O) BioMed Central 